\title{
Prevalência de sobrepeso, obesidade e obesidade abdominal em operadores de duas centrais de atendimento telefônico de São Paulo*
}

\section{Prevalence of overweight and abdominal obesity in operators of two call centers in São Paulo}

\author{
Maria Fernanda Cristofoletti ${ }^{1}$, Maria de Fátima Marinho Souza ${ }^{2}$, \\ Marly Augusto Cardoso ${ }^{3}$, Lys Ester Rocha ${ }^{4}$
}

\begin{abstract}
Cristofoletti MF, Souza MFM, Cardoso MA, Rocha LE. Prevalência de sobrepeso, obesidade e obesidade abdominal em operadores de duas centrais de atendimento telefônico de São Paulo. Saúde, Ética \& Justiça. 2006;11(1/2):19-28.

RESUMO: Objetivo: Avaliar o estado nutricional de operadores de centrais de atendimento telefônico segundo sexo e alteração de peso após começar o trabalho como operador de telemarketing de duas centrais do município de São Paulo. Metodologia: Estudo transversal envolvendo 214 trabalhadores. Utilizou-se o Índice de Massa Corporal (IMC) e a Circunferência da Cintura (CC) para identificar o estado nutricional. Os dados das condições de trabalho foram obtidos com entrevista, observação e aplicação de questionário. O IMC e CC foram descritos em relação ao sexo e alteração de peso após trabalhar como operador de telemarketing utilizando teste de qui-quadrado de Pearson com significância a 5\%. Resultados: A maioria da população era do sexo feminino com idade entre 19 e 39 anos. Para $30,4 \%$ dos operadores a ansiedade do trabalho alterou o consumo alimentar. As mulheres alteravam significantemente a sua alimentação visando à perda de peso. A prevalência de sobrepeso foi de $24,8 \%$ e obesidade $9,3 \%$. Os homens apresentaram maior sobrepeso (35,2\%), obesidade (12,9\%) e obesidade abdominal $(27,8 \%)$. Das condições de trabalho foi observada a postura sentada por todo o período, com uma pausa de 15 minutos para alimentação. $O$ aumento de peso após trabalhar como operador ocorreu para $49 \%$ sendo associado significantemente as maiores prevalências de sobrepeso e obesidade. Conclusão: a prevalência de obesidade e sobrepeso foi alta, predominando entre os homens. Nas condições de trabalho observou-se a presença de trabalho sedentário e fatores de estresse que podem estar contribuindo para estas prevalências.
\end{abstract}

DESCRITORES: Sobrepeso. Obesidade. Telefone/utilização. Pessoal de operação/prevenção \& controle. Condições de trabalho.

\footnotetext{
* Subvencionado pelo Conselho Nacional de Pesquisa e Desenvolvimento Tecnológico (CNPq) - bolsa concedida a mestrandos $-\mathrm{n}$ ㅇ do processo: 131111/02-7. Parte da dissertação de mestrado de autoria de Cristofoletti MF, apresentado ao Departamento de Saúde Ambiental, Faculdade de Saúde Pública - Universidade de São Paulo intitulada "Avaliação do estado nutricional de operadores de telemarketing submetidos a três turnos fixos de trabalho", em 2003.

${ }^{1}$ Universidade de São Paulo - Faculdade de Saúde Pública - Departamento de Saúde Ambiental. Av. Dr. Arnaldo, 715, Cerqueira César, CEP 02146-904, São Paulo - SP.

${ }^{2}$ Ministério da Saúde - Universidade Federal de Cuiabá - Programa de Pós-Graduação em Saúde Coletiva. Shigs 713, bloco y, casa 3, asa sul CEP 70380-725, Brasília - DF.

${ }^{3}$ Universidade de São Paulo - Faculdade de Saúde Pública - Departamento de Nutrição - End: Av. Dr. Arnaldo, 715, Cerqueira César, CEP 02146-904, São Paulo - SP.

${ }^{4}$ Universidade de São Paulo - Faculdade de Medicina - Departamento de Ética Médica, Medicina Legal e Medicina Social e do Trabalho (LIM 40). Rua Teodoro Sampaio, 115, Cerqueira César, CEP 05405-000, São Paulo - SP. Endereço para correspondência: Maria Fernanda Cristofoletti. Rua Cardeal Arcoverde, 500, apto 6, Pinheiros, CEP 05408-000, São Paulo, SP. e-mail: mfcristo@usp.br
} 


\section{INTRODUÇÃO}

A obesidade é um problema de saúde pública mundial, afetando países com diferentes condições sócio-econômicas. No Brasil, os dados da Pesquisa Nacional sobre Saúde e Nutrição - PNSN mostraram que $11,7 \%$ da população feminina e $4,8 \%$ da masculina eram obesos e $29,3 \%$ das mulheres e $25,9 \%$ dos homens apresentavam sobrepeso em 19895 .

Dados mais atuais mostram o avanço do sobrepeso e obesidade no país em homens e a estabilidade da prevalência em mulheres. A última Pesquisa sobre Orçamento Familiar (POF 2002/ 2003) relatou que a obesidade representa $8,9 \%$ do total de homens e $13,1 \%$ das mulheres. O sobrepeso atingia $41,1 \%$ dos homens e $40,0 \%$ das mulheres (IBGE - 2004) $)^{8}$. Na cidade de São Paulo, um estudo descreveu que $13,7 \%$ da população estava obesa $(12,3 \% \text { de homens e } 15,1 \% \text { de mulheres })^{13}$.

Os estudos relacionando condições de trabalho e a prevalência de sobrepeso, obesidade e obesidade abdominal de trabalhadores, em geral, são poucos freqüentes no Brasil. Em um estudo com 647 funcionários de um banco estatal do Rio de Janeiro, verificou-se que a prevalência de sobrepeso era igual a $27,8 \%$ e de obesidade $6,4 \%{ }^{6}$. Outro estudo, com 65 operários de uma empresa metalúrgica do Rio de Janeiro identificou que $44,6 \%$ dos trabalhadores possuíam IMC superior a $25 \mathrm{~kg} / \mathrm{m}^{23}$.

No Brasil, não há investigações sobre prevalência de obesidade em operadores de centrais de atendimento telefônico. Esta atividade tem se expandido de forma expressiva em vários países. As estimativas indicam que o setor emprega cerca de 5 milhões de pessoas nos Estados Unidos e aproximadamente 1,5 milhão na Europa ${ }^{19}$. No Brasil, o número de profissionais envolvidos na atividade foi estimado em cerca de 555 mil em 2005, pela Associação Brasileira de Telemarketing ${ }^{1}$.

No Brasil, a Classificação Brasileira de Ocupações (CBO) define os operadores de centrais de atendimento telefônico como operadores de telemarketing, caracterizando esta profissão pelo atendimento aos usuários, oferecimento de serviços e produtos, prestação de serviços técnicos especializados, entre outras atividades. Os meios de trabalho são formados por telefone (headfone monoauricular) e o computador ${ }^{14}$.

Em face da ausência de estudos na área, fazse necessário investigar o estado nutricional em operadores de centrais de atendimento telefônico. Desta forma, o presente estudo foi desenvolvido com o objetivo de analisar o estado nutricional de operadores de telemarketing em relação ao sexo e alteração de peso devido ao trabalho como operador de telemarketing em duas centrais de atendimento telefônico na cidade de São Paulo - Brasil.

\section{MÉTODOS}

\section{População}

O estudo foi realizado em duas centrais de atendimento telefônico de empresas distintas situadas na zona sul da cidade de São Paulo - SP. A pesquisa foi realizada em duas centrais devido à disponibilidade destas em concordar com a realização do estudo e pela dificuldade em se obter o número total de centrais existentes, pois empresas de diferentes ramos de atividade econômica podem ter este tipo de serviço interna ou externamente à sua estrutura.

A empresa $(A)$ atuava na área de planos de saúde e a outra (B) atendia clientes de uma companhia aérea. As centrais funcionavam ao longo das 24 horas do dia, operando com turnos de seis horas de trabalho que incluíam: manhã, tarde, noite e madrugada. O serviço ocorria também aos sábados, domingos e feriados.

A Central A contava com 115 operadores distribuídos da seguinte forma: turnos matutino e vespertino, 46 operadores cada; turno noturno, 17 operadores; turno da madrugada, 6 operadores. A central B contava com um total de 405 funcionários, dos quais 351 eram operadores. Os operadores do turno da manhã eram 186; os da tarde 124; os da noite 35 e madrugada 6 .

Foi estabelecida uma amostra do total de 520 operadores em atividade nas duas empresas. Para o cálculo do tamanho amostral, utilizou-se a prevalência de obesidade entre mulheres na população do sudeste urbano do Brasil com idade entre 25 e 34 anos $^{5}$. A escolha da prevalência de obesidade de mulheres jovens ocorreu devido ao predomínio de operadores de telemarketing com estas características. O cálculo do tamanho da amostra, considerando os parâmetros: nível de confiança do estudo de 0,95; poder do teste (beta) de 0,90; estimativa de prevalência de obesidade de $24,7 \%$ e número de operadores igual a 520 , resultou em um tamanho amostral de 198 indivíduos. Para evitar possíveis perdas foi acrescentado $10 \%$ ao tamanho amostral, ou seja, 218 indivíduos.

A amostra foi selecionada de forma não aleatória contendo indivíduos dos turnos de trabalho da manhã, tarde e noite. Foram convidados a participar os indivíduos que iniciavam o trabalho nos seguintes horários: entre 6:00 e 8:00 horas (turno matutino); entre 12:00 e 14:00 horas (turno vespertino) e entre 18:00 e 
20:00 horas (turno noturno). Foram excluídos os operadores que estavam de licença saúde e afastados por férias.

No final, participaram do estudo 72 operadores do turno matutino, 97 do turno vespertino e 49 do turno noturno resultando em 218 operadores. Desses três foram excluídas por serem gestantes e outra porque não foi obtido o dado de sua altura. Dos 214 operadores, 83 eram da central A e 131 eram da central B.

O projeto de pesquisa foi aprovado pelo Comitê de Ética em Pesquisa da Faculdade de Saúde Pública da Universidade de São Paulo (COEP-FSP/ USP). Todos os participantes assinaram um Termo de Consentimento livre e esclarecido.

\section{Análise das condições de trabalho}

A análise das condições de trabalho foi efetuada no período entre agosto a novembro de 2001 através de visitas semanais às duas centrais de atendimento, constando das seguintes etapas:

- 12 entrevistas individuais com profissionais da administração e operadores que ocupavam cargos em diferentes setores para conhecimento do processo de trabalho e possíveis repercussões na saúde;

- $\quad$ observação dos trabalhadores em diferentes núcleos e turnos de trabalho.

As entrevistas abordavam o tempo de trabalho na empresa, as funções desempenhadas, as facilidades e dificuldades do trabalho como operador e o tempo para alimentação. As entrevistas duraram cerca de duas horas e as respostas dos operadores foram registradas.

A observação dos operadores verificou a situação de trabalho e a escuta das ligações atendidas pelos operadores. No total, foram realizadas 10 escutas (cinco em cada empresa) durando cerca de 20 horas nos diferentes turnos.

\section{Questionário sobre características sócio- demográficas, hábitos alimentares e variação de peso na vida adulta}

Foi aplicado um questionário contendo dados de características sócio-demográficas (sexo, idade, estado conjugal, paridade e escolaridade), hábito alimentar (número, regularidade quanto ao horário das refeições e modificação da alimentação no último mês). A partir das entrevistas optou-se por incluir duas questões relacionadas à modificação de peso na vida adulta: se a ansiedade do trabalho alterava o consumo alimentar ( $\mathrm{O}$ fato de você não poder responder o cliente o (a) deixa ansioso (a) a ponto de afetar o seu consumo alimentar?) e se houve alteração de peso após trabalhar como operador de telemarketing (Seu peso alterou depois que o Sr. (a) começou trabalhar como operador de telemarketing?). Quando os operadores informaram que alteraram o peso após trabalhar como operador foi respondida uma questão aberta sobre os motivos desse fato.

\section{Medidas antropométricas}

O Índice de Massa Corporal (IMC) foi calculado como a razão entre a medida do peso em quilos e o quadrado da altura em metros $\left(\mathrm{kg} / \mathrm{m}^{2}\right)$. O peso corporal foi avaliado por balança digital com precisão em gramas (Tanita). A altura corporal foi medida através de estadiômetro de parede com precisão 0,1 $\mathrm{cm}$. Considerou-se menor do que $18,5 \mathrm{~kg} / \mathrm{m}^{2}$ baixo peso; entre 18,5 a $24,9 \mathrm{~kg} / \mathrm{m}^{2}$ normal; entre 25,0 e $29,9 \mathrm{~kg} / \mathrm{m}^{2}$ sobrepeso e maior igual a $30,0 \mathrm{~kg} / \mathrm{m}^{2}$ obesos conforme estabelecido pela Organização Mundial da Saúde ${ }^{21}$. Utilizou-se a circunferência da cintura (CC) como indicador de obesidade abdominal sendo a medição efetuada com fita inelástica, adotando-se procedimentos padronizados por Lohman et al. ${ }^{11}$. Em relação à $\mathrm{CC}$, indivíduos com $C C \geq 94$ para homens $\mathrm{e} \geq 80 \mathrm{~cm}$ para mulheres foram classificados com obesidade abdominal seguindo a classificação da $\mathrm{OMS}^{21}$. No total, três medidas de peso corporal, altura e circunferência da cintura foram obtidas para cada participante e foi calculada a média individual.

\section{Análise dos dados}

Primeiro, foi realizada a análise descritiva em freqüências e porcentagens com os dois desfechos de interesse: sexo e alteração de peso após trabalhar como operador. As variáveis independentes utilizadas para a análise foram: idade; estado conjugal, paridade, escolaridade, turno de trabalho, regularidade quanto ao horário das refeições, modificação da alimentação no último mês e presença da ansiedade no trabalho como alterando o consumo alimentar.

Depois foram descritas características das condições de trabalho observadas pelos 
Cristofoletti MF et. al. Prevalência de sobrepeso, obesidade e obesidade abdominal em operadores.

pesquisadores ou que foram relatadas pelos operadores como relacionadas com as alterações de peso, basicamente o trabalho sedentário, o conteúdo e ritmo de trabalho e os fatores geradores de estresse no trabalho.

Para verificar a associação entre estado nutricional e sexo ou alteração de peso após trabalhar como operador de telemarketing foi feito teste de associação pelo qui-quadrado $\left(\mathrm{X}^{2}\right)$. Adotou-se o nível de significância de $5 \%(p<0,05)$.

Os dados foram analisados com o uso do software Stata versão $8.0^{18}$.

\section{RESULTADOS}

\section{Características sócio-demográficas}

Dos 214 operadores estudados a maioria era do sexo feminino $(74,8 \%)$. A população estudada era jovem com idade média igual a 28,9 anos (DP $=7,6$ anos), solteira e com ensino superior incompleto. As características da população, segundo sexo, estão apresentadas na Tabela 1. As mulheres são mais jovens, trabalhando mais no turno da tarde. A maioria dos homens trabalha no turno noturno.

Tabela 1 - Características gerais dos operadores de telemarketing segundo sexo. São Paulo, 2002 ( $n$ =

214)

\begin{tabular}{|c|c|c|c|}
\hline Variáveis & $\begin{array}{c}\text { Masculino }(n=54) \\
n(\%)\end{array}$ & $\begin{array}{c}\text { Feminino }(n=160) \\
n(\%)\end{array}$ & p \\
\hline \multicolumn{4}{|l|}{ Idade } \\
\hline $19-29$ & $16(29,6)$ & $66(41,3)$ & $0.02^{* 1}$ \\
\hline $30-39$ & $31(57,4)$ & $58(36,3)$ & \\
\hline $40-52$ & $7(13,0)$ & $41(22,4)$ & \\
\hline \multicolumn{4}{|l|}{ Estado Conjugal } \\
\hline Solteiro & $41(75,9)$ & $111(69,4)$ & $0,35^{1}$ \\
\hline Casado/vive com companheiro & $13(24,1)$ & $49(30,6)$ & \\
\hline \multicolumn{4}{|l|}{ Paridade } \\
\hline Sim & $43(79,6)$ & $110(68,7)$ & $0,12^{1}$ \\
\hline Não & $11(20,4)$ & $50(31,3)$ & \\
\hline \multicolumn{4}{|l|}{ Escolaridade } \\
\hline Ensino médio completo & $11(20,4)$ & $43(26,8)$ & $0,38^{1}$ \\
\hline Ensino superior incompleto & $28(51,8)$ & $66(41,2)$ & \\
\hline Ensino superior completo/pós & $15(27,8)$ & $51(31,8)$ & \\
\hline \multicolumn{4}{|l|}{ Horário dos turnos } \\
\hline Manhã & $14(25,9)$ & $58(36,3)$ & $0,00 * 1$ \\
\hline Tarde & $19(35,2)$ & $76(47,6)$ & \\
\hline Noite & $21(38,9)$ & $26(16,3)$ & \\
\hline \multicolumn{4}{|l|}{ Regularidade dos horários das refeições } \\
\hline Todos os dias no mesmo horário & $12(22,2)$ & $28(17,5)$ & $0,11^{1}$ \\
\hline Às vezes no mesmo horário & $16(29,6)$ & $73(45,6)$ & \\
\hline Cada dia em horários diferentes & $26(48,1)$ & $59(36,8)$ & \\
\hline Mudou alimentação no último mês ou está fazendo dieta? & $(n=53)$ & $(n=159)$ & \\
\hline Não & $39(73,5)$ & $94(59,1)$ & $0,00 * 2$ \\
\hline Sim, para perda de peso & $9(17,0)$ & $63(39,6)$ & \\
\hline Sim, para ganho de peso & $5(9,4)$ & $2(1,3)$ & \\
\hline A ansiedade do trabalho alterou seu consumo alimentar? & $(n=49)$ & $(n=155)$ & \\
\hline Alterou o consumo alimentar & $11(22,4)$ & $51(32,9)$ & $0,16^{1}$ \\
\hline Não alterou o consumo alimentar & $38(77,6)$ & $104(67,1)$ & \\
\hline
\end{tabular}

${ }^{1}$ Qui Quadrado de Pearson; ${ }^{2}$ Teste Exato de Fisher; * $p<0,05$ 


\section{Avaliação das condições de trabalho}

A observação do trabalho dos operadores revelou que estes permaneciam sentados em todo o período da jornada no Posto de Atendimento (PA). O posto era formado basicamente pelo telefone, computador, mesa e cadeira onde o operador trabalhava em posição sentada realizando movimentos dos braços, mãos e do aparelho fonador. As cadeiras nem sempre eram ajustadas à altura do trabalhador e havia ausência de suporte para os pés.

Quanto à organização do trabalho, foram analisados os seguintes aspectos: o conteúdo da atividade, carga mental e o controle sobre o ritmo do trabalho.

Quanto ao conteúdo das atividades, em ambas as empresas os operadores realizavam atendimento de chamadas; transferência de chamadas para outras pessoas e setores quando necessário; vendas de bens e produtos; recebimento de reclamações e fornecimento de informações para os usuários das empresas.

Quanto à carga mental, as atividades dos operadores, em ambas as empresas, exigiam intensa concentração, devido ao volume de informações a processar, o nível de responsabilidade do trabalho e a grande variedade de solicitações dos clientes.

Quanto ao controle sobre o trabalho, os operadores referiram não ter controle sobre as atividades. Em ambas as empresas eram dadas duas pausas, uma de 15 minutos, onde normalmente os operadores realizavam um lanche e outra de 10 minutos para outras necessidades fisiológicas.

O ritmo de trabalho foi considerado intenso em ambas as empresas, pois o volume de chamadas das centrais cresceu devido à centralização dos atendimentos antes distribuídos por outros locais do Brasil sem o aumento proporcional no número de operadores. Além disso, o ritmo de trabalho imposto pelo sistema automático de distribuição de chamadas tornava fonte importante de tensão por combinar um excessivo número de ligações e a impossibilidade de pequenas pausas entre as chamadas. O maior volume de ligações ocorria no período das 9:00 às 12:00 horas.

Os relacionamentos com os clientes geravam ansiedade pela exigência dos usuários para atendimento rápido, o monitoramento das ligações pela empresa e a solicitação dos supervisores para o atendimento de forma cortês de todos os telefonemas mesmo quando os usuários demonstrassem agressividade.

\section{Hábitos alimentares e alteração de peso após trabalho como operador}

Havia a possibilidade de adquirir alimentos em máquinas de venda nas duas centrais, como salgadinhos, biscoitos, refrigerantes, barra de cereais, balas e chicletes (dietéticos e com açúcar).

A maioria dos operadores fazia de 3 a 4 refeições ao dia. Quanto aos horários das refeições para as mulheres predominou a realização das refeições às vezes no mesmo horário enquanto para os homens cada dia em horários diferentes, mas sem diferenças estatisticamente significantes (Tabela 1).

Quanto aos hábitos alimentares os homens referiram não ter mudado a alimentação no último mês enquanto as mulheres referiram estar fazendo dieta para perda de peso com diferença estatística (Tabela 1).

A ansiedade do trabalho alterando o consumo alimentar foi verificada para $22 \%$ dos homens e $32 \%$ das mulheres sem diferenças estatisticamente significativas (Tabela 1).

O aumento de peso após trabalhar como operador foi referido por $49,0 \%$ dos operadores; $42,8 \%$ não referiram alteração e $8,2 \%$ a diminuição do peso. Em relação ao sexo, $51 \%$ dos homens e $48 \%$ das mulheres aumentaram o peso após o trabalho como operador.

Entre os motivos relatados como relacionados ao aumento de peso após trabalhar como operadores foram: trabalho predominante sedentário, horários de lanches diferentes a cada dia, estresse do trabalho e beliscar alimentos.

Não houve diferença estatisticamente significante em relação à alteração de peso após trabalhar como operador segundo faixas etárias, estado conjugal, paridade, escolaridade e regularidade dos horários das refeições. As diferenças observadas foram em relação ao turno de trabalho, com predomínio do aumento de peso para os operadores do turno da manhã (Tabela 2).

Em relação à mudança de alimentação no último mês, dos que não alteraram a dieta $43,4 \%$ relataram aumento de peso após trabalhar como operador enquanto que entre os trabalhadores que referiram estar fazendo dieta para perda de peso $63 \%$ referiram ter aumentado peso após o trabalho como operador com diferenças estatisticamente significantes (Tabela 2).

A ansiedade do trabalho alterou o consumo alimentar para $68,1 \%$ dos operadores (Tabela 2 ). 
Cristofoletti MF et. al. Prevalência de sobrepeso, obesidade e obesidade abdominal em operadores.

Tabela 2 - Características gerais dos operadores de telemarketing, segundo alteração de peso após iniciar o trabalho como operador de telemarketing. São Paulo, $2002(n=214)$

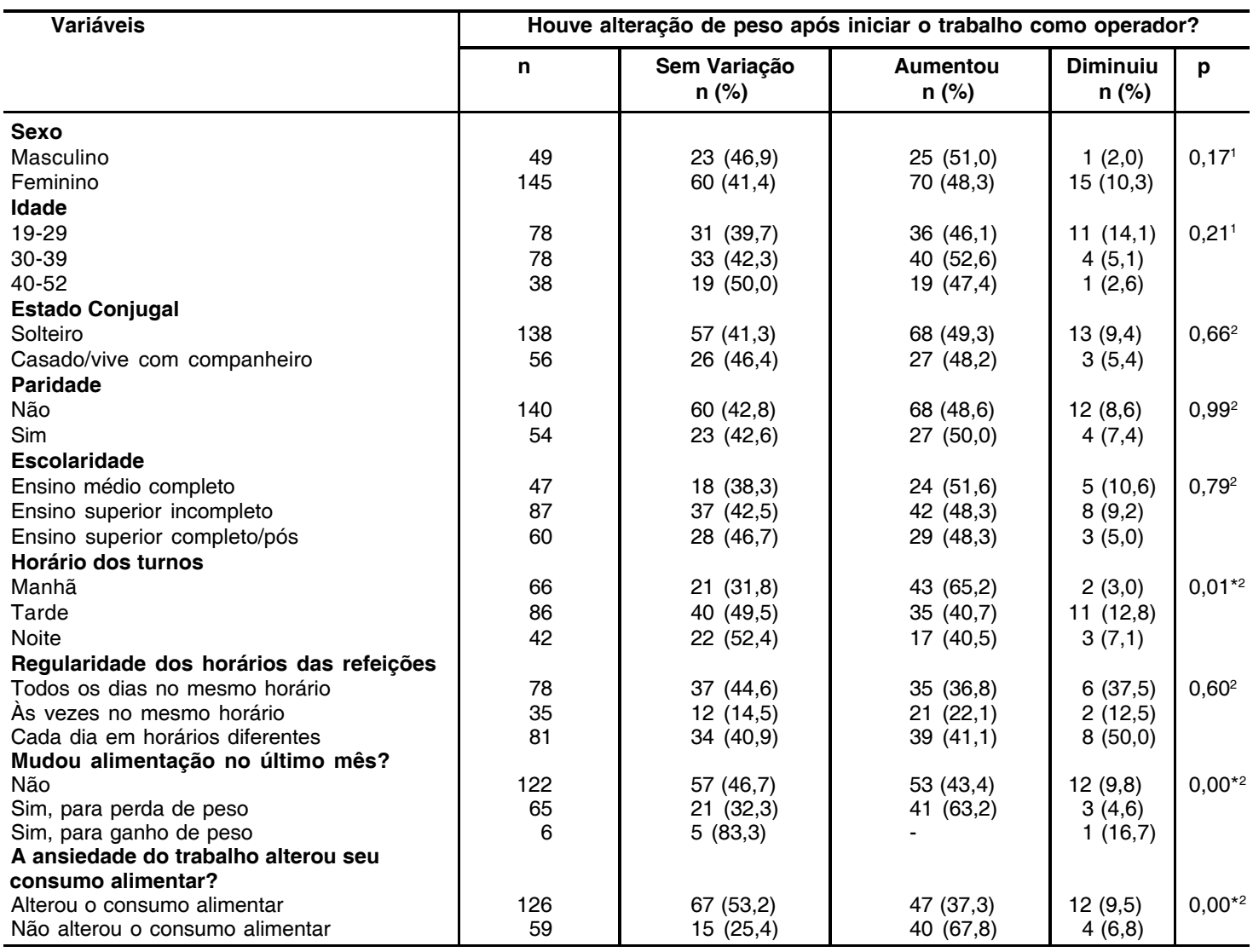

${ }^{1}$ Qui Quadrado de Pearson; ${ }^{2}$ Teste Exato de Fisher; ${ }^{*} \mathrm{p}<0,05$

\section{Avaliação do estado nutricional}

A prevalência de sobrepeso entre os operadores foi de $24,2 \%$ (IC 95\% = 18,9\% - 30,6\%), e de obesidade de $9,3 \%$ (IC $95 \%=5,4 \%-13,3 \%)$. A associação entre sexo e obesidade não apresentou significância estatística, apesar dos homens apresentaram maiores prevalências de obesidade (Tabela 3).

Tabela 3 - Distribuição numérica e percentual da população segundo estado nutricional e sexo em operadores de duas centrais de atendimento telefônico. São Paulo, 2002

\begin{tabular}{|c|c|c|c|c|c|}
\hline & \multicolumn{2}{|c|}{ Homem $(n=54)$} & \multicolumn{2}{|c|}{ Mulheres $(n=160)$} & \multirow[t]{2}{*}{ p } \\
\hline & $\mathrm{n}$ & $\%$ & $\mathrm{n}$ & $\%$ & \\
\hline $\begin{array}{l}\text { Classificação do IMC } \\
\text { Baixo peso }\left(<18,5 \mathrm{~kg} / \mathrm{m}^{2}\right) \\
\text { Normal }\left(18,5-24,9 \mathrm{~kg} / \mathrm{m}^{2}\right) \\
\text { Sobrepeso }\left(25,0-29,9 \mathrm{~kg} / \mathrm{m}^{2}\right) \\
\text { Obeso }\left(=30,0 \mathrm{~kg} / \mathrm{m}^{2}\right) \\
\text { Classificação da CC** } \\
\text { Normal } \\
\text { Obesidade Abdominal }\end{array}$ & $\begin{array}{r}1 \\
27 \\
19 \\
7 \\
\\
39 \\
15 \\
\end{array}$ & $\begin{array}{r}1,9 \\
50,0 \\
35,2 \\
12,9 \\
72,2 \\
27,8\end{array}$ & $\begin{array}{r}8 \\
105 \\
34 \\
13 \\
\\
120 \\
40 \\
\end{array}$ & $\begin{array}{r}5,0 \\
65,6 \\
21,3 \\
8,1 \\
75,0 \\
25,0 \\
\end{array}$ & $0,08^{2}$ \\
\hline
\end{tabular}

${ }^{1}$ Qui Quadrado de Pearson; ${ }^{2}$ Teste Exato de Fisher; * $p<0,05$

** Classificação da CC segundo pontos de corte por risco aumentado para complicações metabólicas decorrentes da deposição de gordura abdominal (homens $=94 \mathrm{~cm}$ e mulheres $=80 \mathrm{~cm}$ ). 
As prevalências nos homens foram: sobrepeso $35,2 \%$ (IC 95\% $=22,0-48,3 \%$ ) e obesidade $13,0 \%$ (IC $95 \%=3,7 \%-22,2 \%)$. Nas mulheres as prevalências de sobrepeso foram 22,3\% (IC 95\% = $14,8 \%$ - $27,6 \%$ ) e obesidade $7,5 \%$ (IC $95 \%=3,3 \%$ $11,6 \%)$

A obesidade abdominal e ocorreu para $26,2 \%$ dos operadores (IC $95 \%=20,2 \%-32,1 \%$ ), sendo maior em homens $27,8 \%$ (IC $95 \%=15,4 \%-40,1 \%$ ) do que a verificada em mulheres $25,0 \%$ (IC 95\% = 18,2 $31,8 \%$ ) sem associação estatística (Tabela 3).

A prevalência de sobrepeso em trabalhadores que referiram ganhar peso após trabalhar como operador foi igual a 31,6\% (IC 95\% 22,05 - 41,09) e de obesos foi igual a 12,6\% (IC 95\% 5,82 - 19,43). A alteração de peso após trabalhar como operador apresentou forte associação com as prevalências de obesidade e não com a obesidade abdominal (Tabela 4).

Tabela 4 - Estado nutricional segundo alteração de peso após trabalhar como operador. São Paulo, 2002

\begin{tabular}{|c|c|c|c|c|}
\hline & \multicolumn{4}{|c|}{ Houve alteração de peso após trabalhar como operador? } \\
\hline & $\begin{array}{l}\text { Sem variação }(n=83) \\
n(\%)\end{array}$ & $\begin{array}{l}\text { Aumentou }(n=95) \\
n(\%)\end{array}$ & $\begin{array}{l}\text { Diminuiu }(n=16) \\
n(\%)\end{array}$ & $\mathbf{p}$ \\
\hline \multicolumn{5}{|l|}{ Classificação do IMC } \\
\hline $\begin{array}{l}\text { Baixo peso }\left(<18,5 \mathrm{~kg} / \mathrm{m}^{2}\right) \\
\text { Normal }\left(18,5-24,9 \mathrm{~kg} / \mathrm{m}^{2}\right)\end{array}$ & $\begin{array}{c}5(6,0) \\
58(69,9)\end{array}$ & $53 \stackrel{-}{(55,8)}$ & $\begin{array}{r}4(44,4) \\
11(68,8)\end{array}$ & $0,00^{1 *}$ \\
\hline Sobrepeso $\left(25,0-29,9 \mathrm{~kg} / \mathrm{m}^{2}\right)$ & $15(18,1)$ & $30(31,6)$ & $1(6,2)$ & \\
\hline Obeso $\left(=30,0 \mathrm{~kg} / \mathrm{m}^{2}\right)$ & $5(6,0)$ & $12(12,6)$ & - & \\
\hline \multicolumn{5}{|l|}{ Classificação da CC** } \\
\hline $\begin{array}{l}\text { Normal } \\
\text { Obesidade Abdominal }\end{array}$ & $\begin{array}{l}65(78,3) \\
18(21,7)\end{array}$ & $\begin{array}{l}66(69,5) \\
29(30,5)\end{array}$ & $\begin{array}{r}14(87,5) \\
2(12,5)\end{array}$ & $0,22^{1}$ \\
\hline
\end{tabular}

${ }^{1}$ Teste Exato de Fisher; ${ }^{*} p<0,05$

** Classificação da CC segundo pontos de corte por risco aumentado para complicações metabólicas decorrentes da deposição de gordura abdominal (homens $=94 \mathrm{~cm}$ e mulheres $=80 \mathrm{~cm}$ )

\section{DISCUSSÃO}

Embora os dados aqui apresentados refiram-se a apenas duas centrais de atendimento telefônico, o presente estudo é importante por ser o primeiro estudo brasileiro sobre o estado nutricional de operadores de centrais de atendimento telefônico, atividade profissional recente e em expansão atualmente.

Observaram-se altas prevalências de sobrepeso e obesidade nos operadores das centrais telefônicas estudadas. Se compararmos estes resultados com os dados da Pesquisa Nacional sobre Saúde e Nutrição (PNSN) de 1989, observa-se que os operadores homens e mulheres apresentaram maiores prevalências de sobrepeso e obesidade nas faixas etárias correspondentes (homens 19-39 anos e mulheres 19-29 anos) 5 .

Neste estudo foram observadas maiores prevalências de sobrepeso e obesidade nos homens. Ao comparar com os dados da última POF (Pesquisa de Orçamento Familiar) de 2002/2003, observa-se que os operadores do sexo masculino apresentaram maior prevalência de obesidade do que a registrada na referida pesquisa para homens do Sudeste (10\%) ${ }^{8}$. Em outra pesquisa, realizada na cidade de São Paulo entre 2001-2002 observou-se prevalência levemente inferior à descrita nesse estudo $(12,3 \%)^{13}$.

As prevalências elevadas de obesidade em homens estão de acordo com a tendência crescente de aumento de sobrepeso e obesidade em homens no Brasil. Ao avaliar as últimas três pesquisas nacionais de 1974, 1989 e 2002/2003, verificou-se que duplicou o percentual de homens com sobrepeso e triplicou o de obesos ${ }^{8}$.

As mulheres apresentaram menores prevalências de sobrepeso e obesidade, alteraram sua alimentação para promover a perda de peso e diminuíram o seu peso na vida adulta. Nas operadoras de telemarketing, a obesidade e o sobrepeso foram menos prevalentes em relação ao descrito na $\mathrm{POF}^{8}$.

A menor prevalência da obesidade nas mulheres pode estar relacionada com a pressão da sociedade para as mulheres manterem-se magras e com dietas saudáveis. Esse estudo encontrou associação estatisticamente significante em relação 
às mulheres que faziam dieta ou mudavam a sua alimentação para perda de peso $(p=0,00)$, o que poderia explicar as menores prevalências de obesidade e sobrepeso em mulheres.

A obesidade abdominal nessa pesquisa não teve associação com o sexo. Em outros estudos foram observadas associações com o fato de serem mulheres $^{9,12}$. Uma possível explicação para essas diferenças, é que muitas mulheres, nessa pesquisa, não possuíam filhos. A obesidade abdominal ocorre em maior proporção nas mulheres com filhos, como mostrou Kac et al. ${ }^{9}$.

A maior prevalência de sobrepeso e obesidade foi associada significativamente à referência de ganho de peso após trabalhar como operador de centrais de atendimento telefônico. Neste estudo verificou-se que no trabalho dos operadores existia a presença constante de postura sentada e de fatores de estresse como o intenso ritmo, a falta de pausas e o relacionamento com os clientes.

Norman et al. ${ }^{15}$ compararam o trabalho de operadores de telemarketing com outras profissões usuárias de computador, verificando o maior tempo de permanência sentado dos operadores. O trabalho em condições sedentárias pode estar contribuindo para o ganho de peso na vida adulta, pois os trabalhadores podem estar comendo mais do que necessitam, produzindo um balanço energético positivo com ganho de peso corporal ${ }^{7}$.

O período no Brasil correspondente ao aumento da obesidade dos homens é o mesmo do crescimento do setor terciário que gerou profissões que despendem pouca energia no trabalho, ou seja, são caracterizadas por serem atividades leves ${ }^{2}$.

Neste estudo outro aspecto verificado nas centrais que podem influenciar nas taxas de sobrepeso e obesidade é a presença de fatores de estresse no trabalho. As tarefas exigiam intensa concentração mental acompanhada de baixo controle dos operadores sobre seu ritmo de trabalho. Os trabalhadores do turno da manhã referiram maior ganho de peso após trabalhar como operador sendo que neste horário ocorre o maior volume de ligações explicando as orientações das empresas para evitarem pausas entre 9:00 e 12:00 horas.

Em relação aos fatores de estresse no trabalho destacamos a falta de autonomia do operador e o intenso controle sobre o seu desempenho seja através do cumprimento de metas quantitativas e qualitativas. Vilela e Assunção ${ }^{20}$ descrevem os mecanismos de controle da atividade em uma central de teleatendimento de empresa de telefonia, categorizando em: controle do tempo, do conteúdo, do comportamento, do volume de serviços realizados e dos resultados, relacionando com o sofrimento mental dos operadores.

A presença de fatores de estresse no trabalho dos operadores também foi verificada no estudo de Raab e Rocha ${ }^{16}$. Esses fatores de estresse poderiam contribuir para o ganho de peso, pois segundo Laitinen et al. ${ }^{10}$ certos indivíduos em situações estressantes utilizariam a alimentação ou bebidas para sentir-se melhor e, a alimentação nessas situações seria rica em açúcares e gorduras.

Uma limitação deste estudo é a coleta da informação da alteração de peso após trabalhar como operador ter sido obtida através de questionário, sem medições antropométricas antes e depois do trabalho na função. Entretanto, dois estudos de validação de peso auto-referido na população brasileira, encontraram alta correlação com o peso auto-referido e o medido. Desta forma, os achados desses estudos sugerem que auto-referir o peso pode providenciar informações confiáveis em estudos epidemiológicos ${ }^{4,17}$. Além disso, pode ter ocorrido um possível viés de seleção pelo fato dos operadores terem sido selecionados de forma não aleatória com a preocupação de incluir diferentes horários de entrada e saída.

\section{CONSIDERAÇÕES FINAIS}

Os resultados evidenciaram alta prevalência de obesidade e sobrepeso entre os operadores de centrais de atendimento telefônico. Verificaram-se maiores prevalências de obesidade associadas nos operadores que referiram aumento de peso após trabalhar nesta função.

A tendência de aumento de sobrepeso e obesidade em homens verificada nas pesquisas sobre nutrição deve ser estudada em relação às atividades profissionais, pois o crescimento do trabalho sedentário pode ter relação com este aspecto, mostrando a importância de serem realizados estudos sobre condições de trabalho e estado nutricional.

Nas Centrais de Atendimento Telefônico é fundamental a inclusão de orientações nutricionais para os operadores nas ações de saúde e segurança no trabalho, visando à prevenção do ganho de peso corporal generalizado e a prevenção de doenças crônicas.

Saúde, Ética \& Justiça, São Paulo. 2006;11(1/2):19-28. 
Cristofoletti MF, Souza MFM, Cardoso MA, Rocha LE. Prevalence of overweight and abdominal obesity in operators of two call centers in São Paulo. Saúde, Ética \& Justiça. 2006;11(1/2):19-28.

ABSTRACTS: Aim: To assess the nutritional status of call center operators according to sex, and weight changes after working as call center operators in two centers in São Paulo. Methods: 214 workers participated in a cross-sectional study. Body Mass Index (BMI) and Waists Circumference (WC) were used to identify nutritional status. Work condition data was collected through interview, work observance and questionnaire. The BMI and the WC were compared with sex, and weight changes using Pearson chi-square. The level of significance was $p<0.05$. Results: Most of the operators were female between the ages of 19-39. Work anxiety changed dietary intake for $30.4 \%$ of call center operators. Women changed their food intake pattern purposefully to lose weight. Overweight was prevalent in $24.8 \%$ and obesity was $9.3 \%$. Men were more overweight $(35.2 \%)$, obese $(12.9 \%)$ and abdominally obese $(27.8 \%)$. Working conditions showed operators working in sitting positions all day having only 15 minutes to eat. Fourty nine percent $(49 \%)$ of operators gained weight after working as call centers operatyors. Working conditions contributed significantily to prevalence of owerweight and obesity. Conclusion: Prevalence of owerweight and obesity in call center operators; mainly in men. Sedentary working conditions and stress factors contributed to prevalence of weight gain.

KEY WORDS: Overweight. Obesity. Operators/prevention\& control. Nutrition. Working conditions. Telephone/utilization.

\section{REFERÊNCIAS}

1. Associação Brasileira de Telemarketing $A B T$ Telemarketing Brazilian Association). O que é telemarketing [citado em 18 fev. 2005]. Disponível em: http://www.abt.org.br/telemarketing.htm.

2. Brasil. Ministério da Saúde. Coordenação de Doenças Cardiovasculares. Doenças cardiovasculares no Brasil - Sistema Único de Saúde (SUS). Brasília: Ministério da Saúde; 1993. p.36.

3. Castro MBT, Anjos LA, Lourenço PM. Padrão dietético e estado nutricional de operários de uma empresa metalúrgica do Rio de Janeiro, Brasil. Cad Saúde Publica (Rio de Janeiro). 2004;20:926-34.

4. Chor D, Coutinho ESF, Laurenti R. Reliability of selfreported weight and height among state bank employee. Rev Saúde Pública (São Paulo). 1999(1):16-23.

5. Coitinho DC, Leão MM, Recine E, Sichieri R. Condições nutricionais da população brasileira: adultos e idosos - pesquisa nacional sobre saúde e nutrição. Brasília: Ministério da Saúde, Instituto Nacional de Alimentação e Nutrição; 1989. p.39.

6. Ell E, Camacho LAB, Chor D. Perfil antropométrico de funcionários de um banco estatal no Estado do Rio de Janeiro/Brasil: I - índice de massa corporal e fatores sócio-demográficos. Cad Saúde Publica (Rio de Janeiro). 1999;(15):113-21.

7. Grandjean E. Manual de ergonomia - adaptando o trabalho ao homem. 4a. ed. Porto Alegre: Bookman; 1998. p. 338.

8. Instituto Brasileiro de Geografia e Estatística (IBGE). Pesquisa de orçamentos familiares - POF 2002/2003. excesso de peso atinge 38,8 milhões de brasileiros adultos [citado em 31 jan. 2004]. Disponível em: http:/ /www.ibge.gov.br/home/presidencia/noticias/ noticia_visualiza.php?id_noticia=278.

9. Kac G, Velásquez-Meléndez G, Coelho MASC. Fatores associados à obesidade abdominal em mulheres em idade reprodutiva. Rev Saúde Pública (São Paulo). 2001;(35):46-51.

10. Laitinen J, Ek E, Sovio U. Stress-related eating and drinking behavior and body mass index and predictors of his behavior. Prev Med (Geneva). 2002;(34):29-39.

11. Lohman TG, Roche AF, Martorell R. Anthropometric standardization reference manual. Illinois: Human Kinetics Books; 1988. p.177.

12. Machado PA, Schieri R. Relação cintura-quadril e fatores da dieta em adultos. Rev de Saúde Pública (São Paulo). 2002;(36):198-204.

13. Marcopito LF, Rodrigues SF, Pacheco MA, Shirassu MM, Goldfeder AJ, Moraes MA. Prevalência de alguns fatores de risco para doenças crônicas na cidade de São Paulo. Rev Saúde Pública (São Paulo). 2005;(39):738-45.

14. Matias AM, Neto AMP, Nascimento AA, Livramento BM, Souza E, Pereira FLS, et al. CBO - Classificação brasileira de ocupações - 4223: operador de telemarketing [citado em 22 abr. 2002]. Disponível em: http://www.mtecbo.gov.br/index.htm.

15. Norman K, Nilsson T, Hagberg M, Tornqvist EW, Toomingas $\mathrm{A}$. Working conditions and health among female and male employees at a call center in Sweden. Am J Industrial Med (New York). 2004;(46):55-6.

16. Raab DM, Rocha LE. Psychosocial aspects of the 
Cristofoletti MF et. al. Prevalência de sobrepeso, obesidade e obesidade abdominal em operadores.

work of female call center operators in a Bank of São Paulo, Brazil. Psyche (São Paulo). 2002;(11):109-20.

17. Silveira EA, Araujo CA, Gigante DP, Barros AJD, Lima MS. Validação do peso e altura referidos para diagnóstico do estado nutricional em uma população de adultos no Sul do Brasil. Cad Saúde Pública (Rio de Janeiro). 2005;(1):235-45.

18. Stata Corporation. Stata (Statistics Data Analysis). Version 6.0. Texas: Stata Corporation; 2000.

19. Toomingas A, Hagman M, Hansson R, Isaksson IA, Kjellberg A, Norman K, Tornquvist E. Symptoms and clinical findings from the musculoskeletal system among operators at a call center in Sweden - a 10 month follow-up study. National Institute for Working Life; 2002.

20. Vilela LVO, Assunção AA. Os mecanismos de controle da atividade no setor de teleatendimento e as queixas de cansaço e esgotamento dos trabalhadores. Cad Saúde Pública (Rio de Janeiro). 2004;20(4):1069-78.

21. World Health Organization (WHO). Obesity: preventing and managing the global epidemic. Geneva: WHO; 2000. (Technical report series 894). 